\title{
Effect of pelletization pressure on structural properties and critical current hysteresis of ceramic superconducting $\mathrm{Bi}_{1.7} \mathrm{~Pb}_{0.3} \mathrm{Sr}_{2} \mathrm{Ca}_{2} \mathrm{Cu}_{3} \mathrm{O}_{y}$
}

\author{
M. Tepe ${ }^{*}, 1$, I. Avcı ${ }^{1,2}$, and D. Abukay ${ }^{2}$ \\ 1 Department of Physics, Faculty of Science, Ege University, 35100 Bornova, Izmir, Turkey \\ 2 Department of Physics, Faculty of Science, Izmir Institute of Technology, 35436 Gülbahçe Campus, \\ Urla, Izmir, Turkey
}

Received 28 November 2002, revised 26 February 2003, accepted 20 March 2003

Published online 14 July 2003

PACS 74.62.Bf, 74.62.Fj, 74.72.Hs

The effect of pelletization pressures on structural properties and critical current hysteresis of $\mathrm{Bi}_{1.7} \mathrm{~Pb}_{0.3} \mathrm{Sr}_{2} \mathrm{Ca}_{2} \mathrm{Cu}_{3} \mathrm{O}_{y}$ samples was investigated. The samples used in this study were prepared by classical solid-state reaction at the pressures from 100 up to $500 \mathrm{MPa}$. The obtained samples were characterized by resistance vs. temperature, $(R-T)$, critical current density vs. applied magnetic field, $\left(J_{\mathrm{c}}-H\right)$, material density vs. pressure, $(\rho-P), \mathrm{XRD}, \mathrm{SEM}$, and EDAX. The results of this study showed that the quality of electrical and structural properties of Bi-2223 bulk superconductors strongly depends on the pelletization pressure. Pressing of bulk samples at $400 \mathrm{MPa}$ produces textured grain alignment and associates microstructural modifications in order to enhance flux pinning and thus increases current carrying capacities.

\section{Introduction}

The superconducting phases of $\mathrm{Bi}-\mathrm{Sr}-\mathrm{Ca}-\mathrm{Cu}-\mathrm{O}$ system are often described by the atomic ratios of the ingredient elements, and by the superconducting transition temperatures. The highest transition temperature of the Bi-2223 is $110 \mathrm{~K}$. So as to obtain a material, which has a homogeneous single phase and a high transition temperature, different methods are used such as doping with $\mathrm{Pb}[1]$ and vanadium atoms [2], adjustment of the thermal treatment $[3,4]$, etc. The structure and current transport in granular superconductors are governed by the nature of the diamagnetic grains and their interconnections, which comprise the superconducting matrix. The size and distribution of the interconnections and their properties greatly depend on the processing method used which enhances the critical current density by growing grains, improving the grain boundary stoichiometry, the intergranular coupling and grain alignment.

The hysteresis in critical current-magnetic field curves was reported for high $T_{\mathrm{c}} \mathrm{Bi}-\mathrm{Sr}-\mathrm{Ca}-\mathrm{Cu}-\mathrm{O}$ superconductors [5]. Transport critical current densities of bulk ceramic high $T_{\mathrm{c}}$ superconductors have generally been restricted to values approximately $10^{3} \mathrm{~A} / \mathrm{cm}^{2}$ at $77 \mathrm{~K}$ in self-field because of weak intergranular current transfer. The critical current density $J_{\mathrm{c}}(B)$ has been elucidated by a model consisting of strongly superconducting grains joined by Josephson junctions [6]. In small magnetic fields critical current densities of bulk samples are rapidly depressed to values less than $10 \mathrm{~A} / \mathrm{cm}^{2}$. The hysteresis in high $T_{\mathrm{c}}$ superconductors has been given by using a bridge model in which edge pinning at the bank becomes a source of hysteresis since the surface currents in the narrow part of the bridge add to or subtract from the

\footnotetext{
${ }^{*}$ Corresponding author: e-mail: tepe@sci.ege.edu.tr, Fax: +90 2323881036
} 
transport current depending on the direction of applied field [7]. Hysteretic behavior of transport critical current was reported by Abukay et al. [8] for $\mathrm{Bi}(\mathrm{Pb})-\mathrm{Sr}-\mathrm{Ca}-\mathrm{Cu}-\mathrm{O}$ system and SEM studies on $\mathrm{Bi}(\mathrm{Pb})-\mathrm{Sr}-\mathrm{Ca}-\mathrm{Cu}-\mathrm{O}$ were reported by Bunescu et al. [11].

The influence of pelletization pressure on magnetic susceptibility of $\mathrm{Bi}(\mathrm{Pb})-\mathrm{Sr}-\mathrm{Ca}-\mathrm{Cu}-\mathrm{O}$ was reported by Fradina et al. [9]. The purpose of this work is to give the effect of pelletization pressure on the structural properties and the critical current hysteresis of Bi-system in the applied magnetic field. We report our results of $R-T, \mathrm{XRD}, \mathrm{SEM}, \mathrm{EDAX}$, and hysteresis measurements on $I_{\mathrm{c}}$ for different samples, which pelletized at the pressures of 100 to $500 \mathrm{MPa}$.

\section{Experiments}

The bulk ceramic samples used in this study were prepared by the ordinary process using $\mathrm{Bi}_{2} \mathrm{O}_{3}, \mathrm{PbO}$, $\mathrm{SrCO}_{3}, \mathrm{CaCO}_{3}$, and $\mathrm{CuO}$ powders. The powder of these oxides and carbonates were mixed by molar ratio 1.7:0.3:2:2:3 to obtain $\mathrm{Bi}_{1.7} \mathrm{~Pb}_{0.3} \mathrm{Sr}_{2} \mathrm{Ca}_{2} \mathrm{Cu}_{3} \mathrm{O}_{y}$ nominal composition. After mechanically mixing in an agat mortar the final mixture in powder form was calcined twice at $800{ }^{\circ} \mathrm{C}$ for $24 \mathrm{~h}$. The calcined material was ground and pressed into pellets of $13 \mathrm{~mm}$ in diameter at pressures from 100 to $500 \mathrm{MPa}$. Then the pellets were sintered at $840{ }^{\circ} \mathrm{C}$ for $200 \mathrm{~h}$ with three intermediate grindings and the cooled to room temperature at a rate of $4{ }^{\circ} \mathrm{C} / \mathrm{min}$.

The bulk samples for $I_{\mathrm{c}}$ vs. $H$ measurements were cut into rectangular bars from the pellets and they were tinned by using abrasive papers. The $I_{\mathrm{c}}$ vs. $H$ measurements were made using a standard four-probe method, and contacts were made using silver paste. In order to contact heating, all measurements were made with the samples immersed in liquid nitrogen $(77 \mathrm{~K})$. For low magnetic fields a copper wire solenoid was used. The field direction was kept perpendicular to the measuring current direction. The critical current densities, $J_{\mathrm{c}}$, were resistively measured in zero field cooled (ZFC) condition where the samples were cooled from room temperature down to $77 \mathrm{~K}$ at zero-magnetic field (neglecting the effect of earth's magnetic field). The critical current was measured in an increasing field, $H_{\mathrm{a}}$, up to a maximum field, $H_{\mathrm{m}}$. The applied field, $H_{\mathrm{a}}$, was decreased gradually down to $-H_{\mathrm{m}}$. The critical current, $I_{\mathrm{c}}$, was defined by taking a voltage drop of $1 \mu \mathrm{V} / \mathrm{cm}$ across the voltage terminals.

The ac electrical resistance measurements were made by using standard four-probe method by a two phase SR-530 lock-in amplifier. The zero-resistance temperature, $T_{\mathrm{c} 0}$, was obtained from $R(T)$ curves. X-ray diffraction patterns of the samples were obtained with Rigau D. Max 3 CXRD by using monochromatic $\mathrm{CuK}_{\alpha}$ radiation in the $2 \Theta=3^{\circ}$ to $60^{\circ}$ range. SEM photographs for the study of surface morphology of the samples and EDAX analysis were obtained using a XL30S FEG SEM.

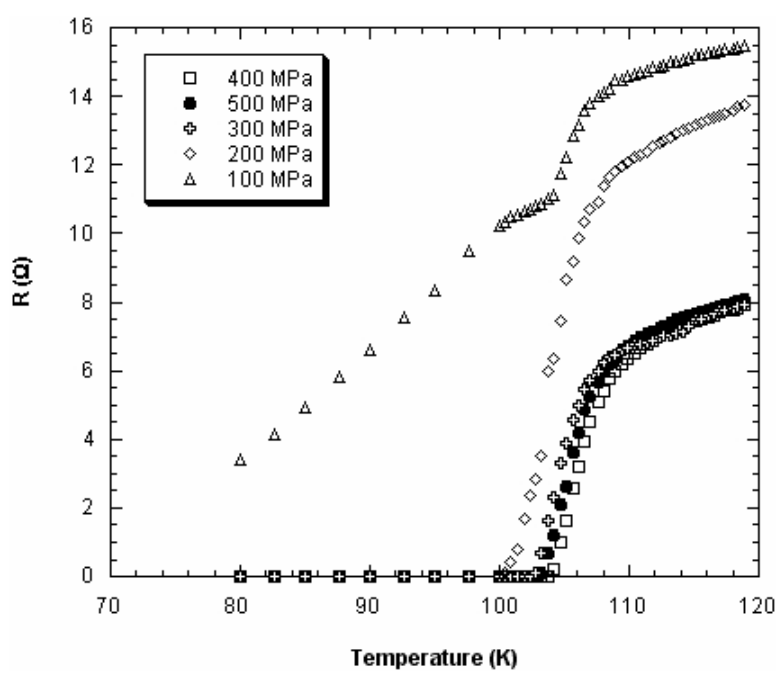

Fig. 1 Resistance vs. temperature curves of samples pelletized at different pressures from 100 up to $500 \mathrm{MPa}$. 

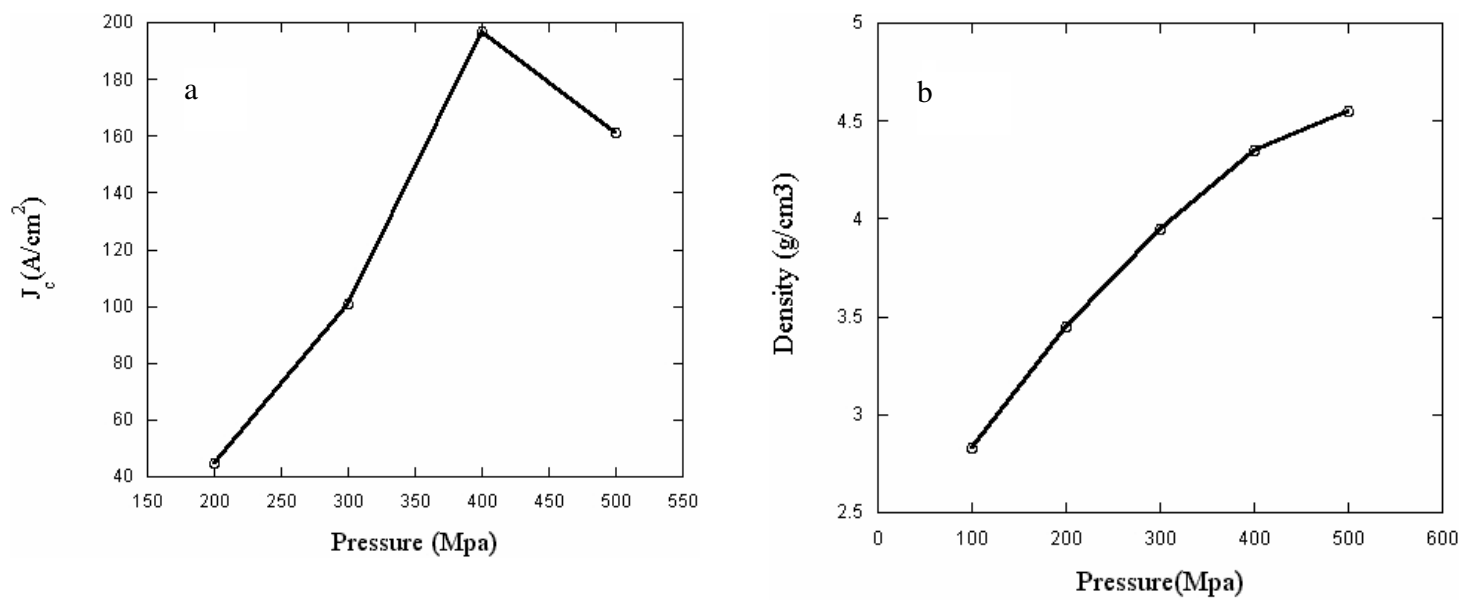

Fig. 2 a) Critical current density vs. pressure at $77 \mathrm{~K}$, b) density vs. pressure.
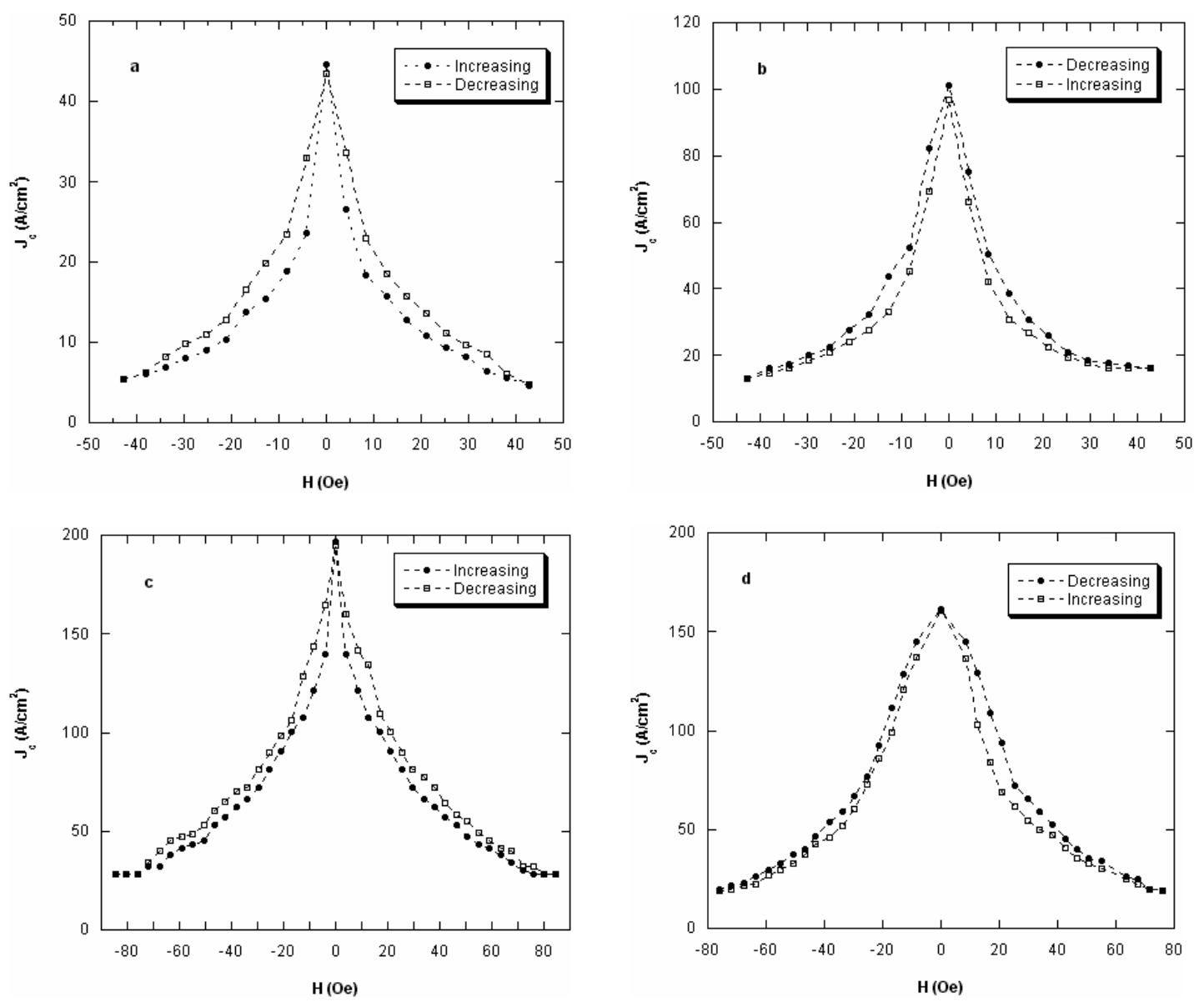

Fig. 3 Hysteresis curves of critical current densities under the decreasing and increasing applied magnetic field. Denoted a), b), c), d) are representing the different samples pelletized at pressures of 200, 300, 400, and $500 \mathrm{MPa}$, respectively. 


\section{Results and discussion}

Figure 1 illustrates the resistance of the $\mathrm{Bi}_{1.7} \mathrm{~Pb}_{0.3} \mathrm{Sr}_{2} \mathrm{Ca}_{2} \mathrm{Cu}_{3} \mathrm{O}_{y}$ samples as a function of temperature, $R(T)$. The resistance of the samples touched down to zero in the range of 100 to $105 \mathrm{~K}$ depending on their pelletization pressures. For the sample, which pelletized at $100 \mathrm{MPa}$, as seen in Fig. 1, the resistance did not approach zero because of its highly porous structure. For samples, which pelletized at 300, 400, and $500 \mathrm{MPa}$, their normal state resistances above the transition temperature were small compared to the other two, and also for these three samples, the transition temperatures were almost the same.

Figure 2 a illustrates the zero-field critical current density of samples versus pressure, $\left(J_{\mathrm{c}}(0)-P\right)$. As can be seen in this figure, critical current density increases with the increasing pressure, and reaches its maximum value at $400 \mathrm{MPa}$, then decreases to a lower value while the pressure rises up to $500 \mathrm{MPa}$. Figure $2 \mathrm{~b}$ represents the density of the samples as a function of pressure, $(\rho-P)$. With increasing pressure from 100 to $500 \mathrm{MPa}$, the density of the samples monotonously increased from 2.9 to $4.5 \mathrm{~g} / \mathrm{cm}^{3}$.

Figure 3 shows the systematic of the hysteretic response of the transport critical current densities, $J_{\mathrm{c}}$, of the samples as a function of applied field, $H_{\mathrm{a}}$, in liquid nitrogen temperature $(77 \mathrm{~K})$. In Fig. 3a, the sample, which pelletized at $200 \mathrm{MPa}$, yields the zero field critical current density of about $45 \mathrm{~A} / \mathrm{cm}^{2}$. The critical current density for the virgin curve drops steeply with increasing field. This is a typical behavior for the ceramic bulk superconductors and has been given a detailed analysis by Peterson and Ekin [6]. The value of $J_{\mathrm{c}}$ noticeably differs from the virgin curve by increasing as the field sweeps in the decreasing direction. The same effect was observed for the negative sweep of the field. The maximum fields at both positive and negative directions were up to 50 Oe for this sample. $J_{\mathrm{c}}$ values were measured for initially increasing field towards positive direction. When the field was decreased from a positive maximum $H_{\mathrm{m}}$, hysteresis showed up. The same effect was observed for the negative sweep of the field. The main feature in this behavior is the depression of the maximum value of the critical current density. This shows us a clear hysteretic behavior in $J_{\mathrm{c}}$. In Fig. $3 \mathrm{~b}$, the sample, which pelletized at $300 \mathrm{MPa}$, shows the zero field critical current density at about $100 \mathrm{~A} / \mathrm{cm}^{2}$. As indicated in this figure, the $J_{\mathrm{c}}$ value at zero-field was increased about two times compared with the first one, and the hysteresis loses for this sample ex-

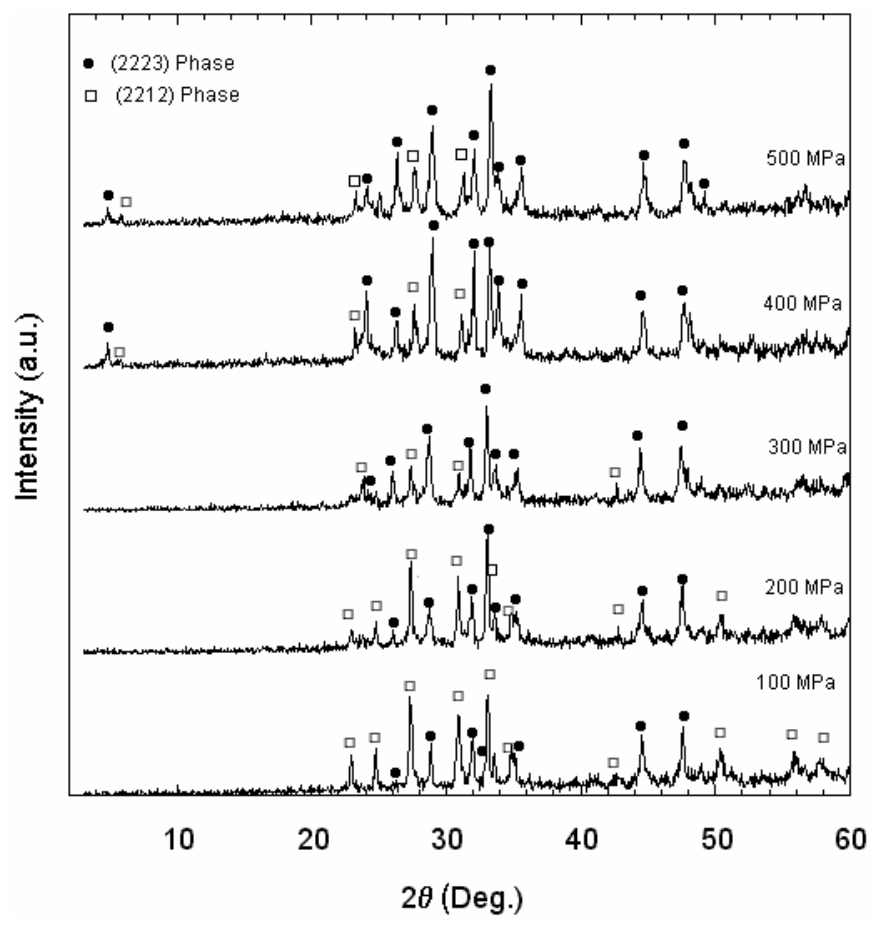

Fig. 4 XRD patterns of the samples pelletized at different pressures of 100, 200,300, 400, and $500 \mathrm{MPa}$. 
isted up to a value of 50 Oe for the maximum value of applied magnetic field, $H_{\mathrm{m}}$. In Fig. $3 \mathrm{c}$, the sample shows the zero field critical current density at about $200 \mathrm{~A} / \mathrm{cm}^{2}$, which was pelletized at $400 \mathrm{MPa}$. The $J_{\mathrm{c}}$ value at zero-field increased up to a maximum value depending on the increasing pressure, and the hysteresis loses existed up to 80 Oe as the maximum value of applied magnetic field, $H_{\mathrm{m}}$. In Fig. $3 \mathrm{~d}$, the curve shows the hysteretic behavior of the value $J_{\mathrm{c}}$ of the sample pelletized at $500 \mathrm{MPa}$, in which, hysteresis loses were observed up to $70 \mathrm{Oe}$, and zero magnetic field current density was depressed to a value of $160 \mathrm{~A} / \mathrm{cm}^{2}$.

The results in Fig. 3 show us the zero-field critical current density, $J_{\mathrm{c}}$, and maximum field, $H_{\mathrm{m}}$, increase with the increasing pelletization pressure from 200 up to $400 \mathrm{MPa}$ for ceramic $\mathrm{Bi}_{1.7} \mathrm{~Pb}_{0.3} \mathrm{Sr}_{2} \mathrm{Ca}_{2} \mathrm{Cu}_{3} \mathrm{O}_{y}$ superconductors and again tend to a lower value at $500 \mathrm{MPa}$. This means that the pelletization pressure plays an important role in texturing mechanism and the quality of intergranular parts of the samples. When the pressure exceeds $400 \mathrm{MPa}$, as can be seen in the SEM micrographs in Fig. 5e, some micro-cracks and defects are generated in the material, and also interconnections of the grains become thinner for the $500 \mathrm{MPa}$ sample. This causes the low $J_{\mathrm{c}}$ compared with that of $400 \mathrm{MPa}$.

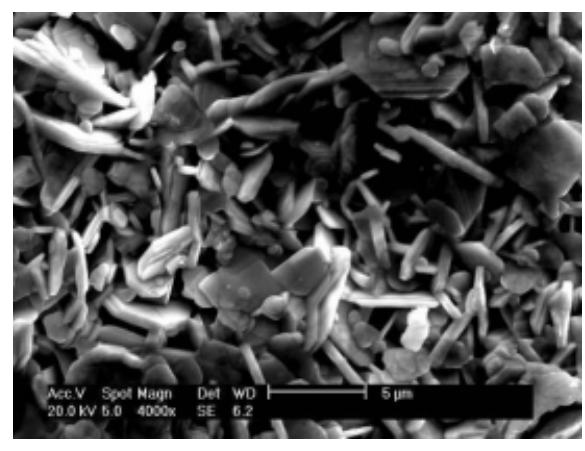

a)

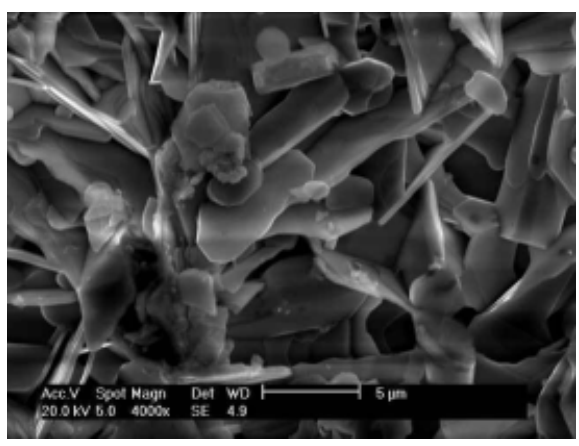

c)

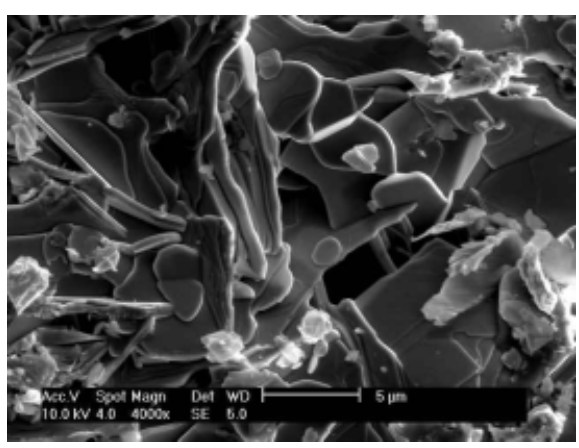

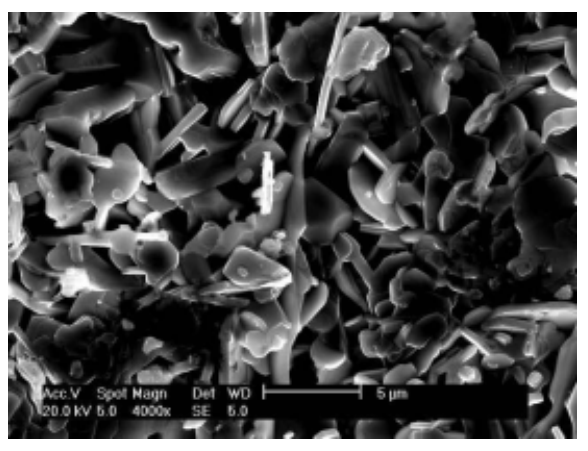

b)

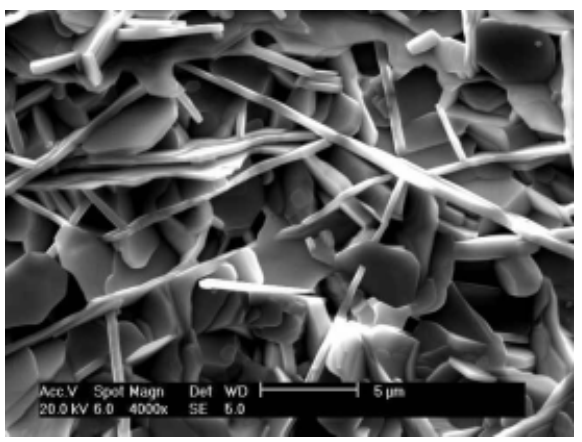

d)

Fig. 5 SEM micrographs of the samples pelletized at different pressures; a), b), c), d), e) are representing the different samples pelletized at pressures of 100, 200, 300, 400 , and $500 \mathrm{MPa}$ respectively. 


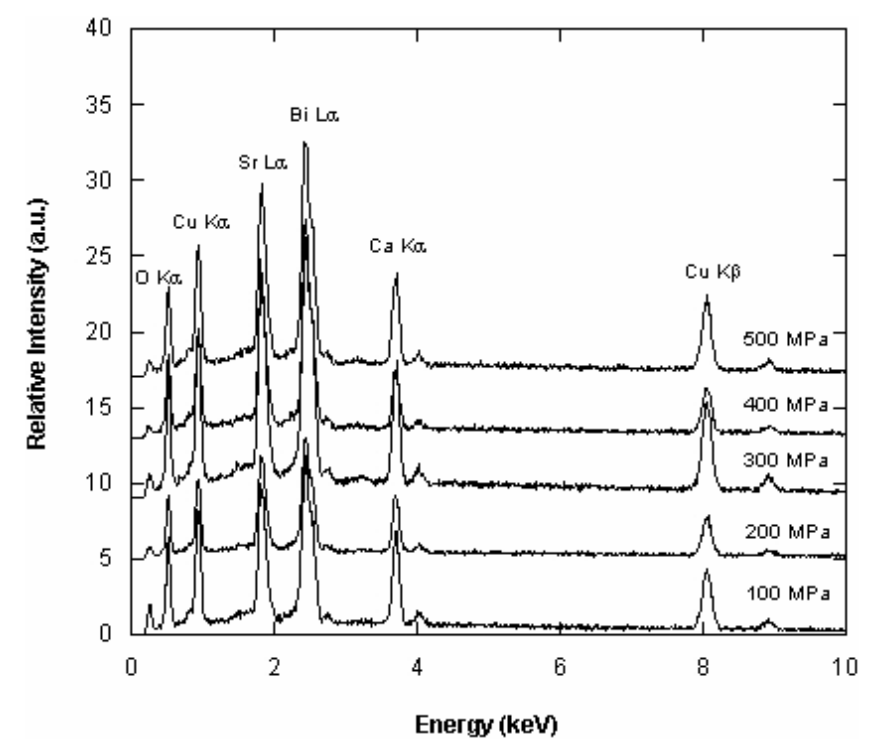

Fig. 6 Typical elemental composition of plate-like crystals of samples pelletized at pressures from 100 up to $500 \mathrm{MPa}$ determined by EDAX.

XRD patterns of pellets at $840{ }^{\circ} \mathrm{C}$ for various pelletization pressures are shown in Fig. 4 . The pressure values of 100, 200, 300, 400, and $500 \mathrm{MPa}$ are given inside of the figure at the different curves. These patterns clearly indicate the related changes in pelletization pressure. It is obvious that the sample pelletized at $100 \mathrm{MPa}$ shows the dominant 2212 low $T_{\mathrm{c}}$ phase. The increasing pressure up to $400 \mathrm{MPa}$ indicates the samples tending to a further formation of 2223 phase, the disappearance of nonsuperconducting phases and the sharpening of reflections, which is presumably related to the elimination or decrease of plane defects induced by incomplete crystallization treatment. At $400 \mathrm{MPa}$, the characteristic (002) peak of the 2223 phase was detectable. Moreover, the enhancement to the growth of the 2223 phase can be found in this sample. As the pressure was increased to $500 \mathrm{MPa}$, the intensity of the peak of 2223 phase decreased compared to that of the sample pelletized at $400 \mathrm{MPa}$. This result suggests that in obtaining the 2223 phase in pelletized bulk samples an optimum pelletization pressure is existing and its value appears to be around $400 \mathrm{MPa}$ as seen in this work.

The SEM study of the samples revealed the microstructures over the entire samples area and representative micrographs of the fractured surfaces of $\mathrm{Bi}-2223$ pellets are shown in Fig. 5. The comparison of micrographs related to the pressures from 100 to $500 \mathrm{MPa}$ clearly indicates that the grains have well defined plate-like shape and are closely packed with little porosity for the sample pelletized at $400 \mathrm{MPa}$. The closely packed grains are indicative of better density of this sample. In Fig. 5a, for the sample, which pelletized at $100 \mathrm{MPa}$, most of the microstructure consisted of flakes with a few plate-like crystals. The amount of plate-like crystals and grain sizes increased with increasing pelletization pressure. As seen in Fig. 5d, the microstructures mainly consisted of the plate-like crystals with a few flakes. This morphology improves the density of the material and the superconducting properties by increasing the contacts between the individual grains. In Fig. 5e, the microstructures consisted of the plate-like crystals with an increasing number of some particles, which can be defined as micro-cracks and defects in the plate-like crystals.

The typical elemental compositions of the plate-like crystals of all samples analyzed by EDAX are shown in Fig. 6. These results are similar to those reported earlier by Takano et al. [1] and Kijima et al. [10].

\section{Conclusion}

In this study, the effects of some texturing mechanisms such as the pelletization pressure on the structural properties, transition temperature, and critical current hysteresis of the Bi-2223 system were inves- 
tigated. The samples used in this study were prepared by classical solid-state reaction at the pressures from 100 up to $500 \mathrm{MPa}$. The obtained samples were characterized by resistance-temperature, critical current density-magnetic field, density-pressure, XRD, SEM, and EDAX. The results of this study showed that the quality of electrical and structural properties of Bi-2223 bulk superconductors strongly depend on the pelletization pressure. $R-T$ and $J_{\mathrm{c}}-H$ measurements showed that the quality of electrical and structural properties of samples decrease while the pressure exceeds $400 \mathrm{MPa}$, which is seen for the sample pelletized at $500 \mathrm{MPa}$. SEM and XRD results found are also supporting these observations. In particular, pressing of bulk samples at $400 \mathrm{MPa}$ produces textured grain alignment and associates microstructural modifications in order to enhance flux pinning and thus increase current carrying capacities.

Acknowledgement The authors would like to express their gratitude to specialists Gökhan Erdoğan and Duygu Oğuz for their valuable suggestions in the analysis of SEM and XRD at the Material Research Center at Izmir Institute of Technology.

\section{References}

[1] M. Takano, J. Takada, K. Oda, H. Kitaguchi, Y. Ikeda, Y. Tomii, and H Mazaki, Jpn. J. Appl. Phys. 27, L1041 (1988).

[2] M. Tepe and D. Abukay, Solid State Commun. 108, 613 (1998).

[3] A. Ono, Jpn. J. Appl. Phys. 27, L2276 (1988).

[4] U. Endo, S. Koyama, and T. Kawai, Jpn. J. Appl. Phys. 27, L1476 (1998).

[5] T. Yamamoto and T. Aomine, Jpn. J. Appl. Phys. 27, L1917 (1988).

[6] R. L. Peterson and J. W. Ekin, Physica C 157, 325 (1989).

[7] E. Tanaka, S. Yamasaki, K. Tani, and A. Yonekura, J. Low Temp. Phys. 74, 263 (1989).

[8] D. Abukay, H. Nakano, and L. Rinderer, Solid State Commun. 79, 573 (1991).

[9] I. A. Fradina, A. F. Alekseev, T. J. Gridasova, V. V. Morozov, and D. O. Jurchenko, Physica C 311, 81 (1999).

[10] N. Kijima, H. Endo, J. Tsuchiya, A. Sumiyama, M. Mizunmo, and Y. Oguri, Jpn. J. Appl. Phys. 27, L1852 (1988).

[11] M. C. Bunescu, G. Aldica, P. Badica, F. Vasiliu, P. Nita, and S. Mandache, Physica C 281, 191 (1997). 